\title{
SELETIVIDADE DE INSETICIDAS A Trichogramma pretiosum (HYMENOPTERA: TRICHOGRAMMATIDAE) COLETADO EM OVOS DE LAGARTA-ENROLADEIRA Bonagota salubricola (Meyrick, 1937) (LEPIDOPTERA: TORTRICIDAE) EM FRAIBURGO, BRASIL
}

\section{SELETIVITY OF PHYTOSSANITARY PRODUCTS TO Trichogramma pretiosum (HYMENOPTERA: TRICHOGRAMMATIDAE) COLLECTED IN APPLE LEAFROLLER EGGS, Bonagota salubricola (Meyrick, 1937) (LEPIDOPTERA: TORTRICIDAE) IN FRAIBURGO, SANTA CATARINA STATE, BRAZIL}

\author{
Emily Silva ARAUJO ${ }^{1}$ \\ Daiane Porto TARGÃO ${ }^{1}$ \\ Patrik Luiz PASTORI ${ }^{2}$ \\ Lino Bittencourt MONTEIRO ${ }^{3}$
}

\begin{abstract}
RESUMO
Neste trabalho foi avaliado o efeito de nove produtos fitossanitários utilizados no controle de pragas de macieiras em Fraiburgo (Brasil) sobre Trichogramma pretiosum Riley 1879 (Hymenoptera: Trichogrammatidae) coletado em ovos de Bonagota salubricola (Meyrick,1937) (Lepidoptera: Tortricidae). Para a realização dos bioensaios foram confeccionadas cartelas $(2,5 \times 0,5 \mathrm{~cm})$ e colados $40 \pm 2$ ovos de Sitotroga cereallela (Oliv., 1819) (Lepidoptera: Gelechiidae), com idade inferior a $24 \mathrm{~h}$ e imersas nas caldas químicas durante cinco segundos. Os adultos do parasitóide foram expostos por 24 horas a resíduos secos de abamectin, clorpyrifos, carbaryl, fenitrothion, malathion, methidathion, phosmet, spirodiclofen e tebufenozide. O parasitismo, a emergência e a razão sexual foram avaliados. O delineamento experimental foi inteiramente casualizado com 10 tratamentos e 25 repetições. Clorpyrifos $(150 \mathrm{~mL} / 100 \mathrm{~L})$ foi classificado como nocivo, malathion (200 $\mathrm{mL} / 100 \mathrm{~L})$ e carbaryl $(360 \mathrm{~mL} / 100 \mathrm{~L})$ se mostraram moderadamente nocivo, fenitrothion $(150 \mathrm{~mL} / 100 \mathrm{~L})$, phosmet (200 $\mathrm{mL} / 100 \mathrm{~L})$, methidathion $(100 \mathrm{~mL} / 100 \mathrm{~L})$ e abamectin $(100 \mathrm{~mL} / 100 \mathrm{~L})$ foram levemente nocivo e tebufenozide $(90 \mathrm{~mL} / 100 \mathrm{~L}) \mathrm{e}$ spirodiclofen $(25 \mathrm{~mL} / 100 \mathrm{~L})$ foram inócuos. A razão sexual não foi afetada. Conclui-se que tebufenozide e spirodiclofen são os produtos fitossanitários que tiveram o menor impacto sobre o parasitismo de $T$. pretiosum e carbaril, methidation e abamectin tiveram o maior impacto sobre a emergência de T. pretiosum.

Palavras chave: Lagarta-enroladeira-da-maçã; macieira; parasitóide de ovos; seletividade.
\end{abstract}

\begin{abstract}
In this work, was evaluated the effect of nine phytossanitary products used in the Apple Integrated Production, to Trichogramma pretiosum Riley 1879 (Hymenoptera: Trichogrammatidae) collected in Bonagota salubricola (Meyrick,1937) (Lepidoptera: Tortricidae). For the bioassay was confectioned paper cards of $2.5 \times 0.5 \mathrm{~cm}$, in which were glued $40 \pm 2$ eggs of Sitotroga cereallela (Oliv., 1819) (Lepidoptera, Gelechiidae) 24 hours old and immersing in the chemical solutions for five seconds. The adults of the parasitoid were exposed to dry residues of the abamectin, clorpyrifos, carbaryl, fenitrothion, malathion, methidathion, phomet, spirodiclofen e tebufenozide, allowing the parasitism for 24 hours. The parasitism rate, adult emergency and sexual ratio was evaluated. The experimental design was entirely randomized with ten treatments and 25 repetitions. Clorpyrifos $(150 \mathrm{~mL} / 100 \mathrm{~L})$ was harmful, malathion $(200 \mathrm{~mL} / 100 \mathrm{~L})$ and carbaryl $(360 \mathrm{~mL} / 100 \mathrm{~L})$ were moderately harmful. Fenitrothion $(150 \mathrm{~mL} / 100 \mathrm{~L})$, phosmet $(200 \mathrm{~mL} / 100 \mathrm{~L})$, methidathion $(100 \mathrm{~mL} / 100 \mathrm{~L})$ and abamectin $(100 \mathrm{~mL} / 100 \mathrm{~L})$ were slightly harmful and tebufenozide $(90 \mathrm{~mL} / 100 \mathrm{~L})$ and spirodiclofen $(25 \mathrm{~mL} / 100 \mathrm{~L})$ were harmless. The sex ratio was not affected. We conclude that tebufenozide and spirodiclofen are the pesticides that had the smallest impact on parasitism and carbaril, methidation and abamectin greater impact on the emergence of $T$. pretiosum.
\end{abstract}

\footnotetext{
${ }^{1}$ Laboratório de Manejo Integrado de Pragas, Departamento de Fitotecnia e fitossanitarismo, Universidade Federal do Paraná, Rua dos Funcionários, 1540, Curitiba-PR, Brasil. emiagro@yahoo.com.br, daia_agro@yahoo.com.br

2 Departamento de Fitotecnia, Universidade Federal do Ceará, Av. Mister Hull, 2977, CEP 60.356-00, Fortaleza-CE, Brasil. plpastori@ufc.br ${ }^{3} \mathrm{Eng}^{\circ}$. Agr ${ }^{\circ}$. Professor, Doutor, Departamento de Fitotecnia e Fitossanitarismo, Universidade Federal do Paraná. Rua dos Funcionários, 1540 , CEP 80035-050, Curitiba-PR, Brasil. E-mail: Ibmonteiro@terra.com.br
} 
ARAÚJO, E.S. et al. Seletividade de inseticidas...

INTRODUÇÃO

A ocorrência de Trichogramma pretiosum Riley, 1879 (Hymenoptera: Trichogrammatidae) foi observada em ovos de lagarta-enroladeira-damaçã, Bonagota salubricola (Meyrick, 1937) (Lepidoptera: Tortricidae) (Monteiro et al., 2004) em pomares de macieira na região de Fraiburgo (Brasil). O parasitismo de $22 \%$ coincide com a eliminação dos inseticidas azynphos methyl, deltametrina, fenthion e parathion em pomares associados ao programa de controle biológico inoculativo do ácaro vermelho (Monteiro, 2001a, b).

A frequência de inseticidas para controle de lagartas e moscas-das-frutas é elevado (Kovaleski 2004), apesar do emprego da estratégia de confusão sexual para tortricídeos (Pastori et al., 2008). Esta situação nos pomares pode prejudicar a abundância de inimigos naturais e o equilíbrio com a praga por modificação fisiológica e comportamental. Os efeitos deletérios de inseticidas utilizados em pomares de macieira no Sul do Brasil, sobre T. pretiosum foram reportados por Giolo et al. (2005) e Manzoni et al. (2006ab, 2007), entretanto as populações testadas não foram coletadas na cultura. Produtos fitossanitários são altamente tóxicos a Trichogramma spp. causando morte devido ao contato com o tóxico. O efeito tóxico pode persistir e ter consequência sobre a performace do parasitoide, mas também ocorrem variações inter e intraespecífica na preferência por hospedeiros e responde às condições ambientais (Bull \& Coleman, 1985; Hassan, 1997; Pratissoli et al., 2003). Assim, linhagens tolerantes a inseticidas podem ser selecionadas em altas pressões de produtos fitossanitários (Baker \& Thorne, 1995). As populações mais eficientes em programas de controle biológico são aquelas coletadas sobre a praga alvo e cultura (Hassan, 1997). Por esse motivo, recomenda-se que os parasitoides usados nos estudos de seletividade sejam coletados sobre a praga e cultura alvo.

O objetivo deste estudo foi avaliar o efeito de nove produtos fitossanitários sobre $T$. pretiosum, linhagem coletada em pomares de macieira em ovos de B. salubricola, em Fraiburgo, Santa Catarina, Brasil.

\section{MATERIAL E MÉTODOS}

Coleta, manutenção e multiplicação dos parasitoides. T. pretiosum linhagem bonagota foi coletada em ovos de $B$. salubricola em pomares comerciais de macieiras em Fraiburgo, SC, Brasil $\left(27^{\circ} 02^{\prime} \mathrm{S}\right.$; $50^{\circ} 55^{\prime} \mathrm{W}$ ) (Monteiro et al. 2004). A multiplicação dos parasitóides ocorreu em ovos de Sitotroga cerealella (Olivier, 1819) (Lepidoptera: Gelechiidae), colados com goma arábica $(25 \%)$ em cartolina azul celeste $(8,0 \times 2,0 \mathrm{~cm})$. As cartelas foram inseridas em tubos de vidro $(8,5 \times 2,5 \mathrm{~cm})$ por 24 horas para o parasitismo de $T$. pretiosum. Os tubos foram transferidos para câmara climatizada à $25 \pm 1^{\circ} \mathrm{C}, 70$ $\pm 10 \%$ rh e 14:10 L:D (Pastori et al., 2007). As colônias de criação receberam introduções de novos parasitóides coletados em ovos de $B$. salubricola a cada ano.

Critério de escolha dos produtos fitossanitários utilizados. Utilizaram-se produtos autorizados pelo Comitê Técnico da Produção Integrada de Maçãs (CTPIM) (Protas \& Sanhueza, 2004). Selecionaramse sete entre dez inseticidas recomendados: carbaryl (Sevin $® 480$ SC, $360 \mathrm{~mL} / 100 \mathrm{~L}$ ), clorpyrifos (Lorsban ${ }^{8} 480$ CE, $150 \mathrm{~mL} / 100 \mathrm{~L}$ ), fenitrothion (Sumithion $® 500 \mathrm{CE}, 150 \mathrm{~mL} / 100 \mathrm{~L}$ ), malathion (Malathion $® 1000 \mathrm{CE}, 200 \mathrm{~mL} / 100 \mathrm{~L}$ ), methidathion (Supracid® $400 \mathrm{CE}, 100 \mathrm{~mL} / 100 \mathrm{~L}$ ), phosmet (Imidan ${ }^{\circledR}$ 500PM, $200 \mathrm{~g} / 100 \mathrm{~L}$ ) e tebufenozide (Mimic ${ }^{\circledR} 240$ SC, $90 \mathrm{~mL} / 100 \mathrm{~L}$ ); dois acaricidas entre quatro: abamectin (Abamectin Nortox $®$ EC, 100 $\mathrm{mL} / 100 \mathrm{~L}$ ) e spirodiclofen (Envidor 240 SC, 25 $\mathrm{mL} / 100 \mathrm{~L})$.

Bioensaios. Ovos de S. cerealella $(40 \pm 2) \mathrm{com}$ 12 horas de desenvolvimento embrionário foram colados em cartelas $(0,5 \times 2,5 \mathrm{~cm})$ de cartolina azul Estas cartelas foram imersas por cinco segundos nas caldas dos produtos fitossanitários, de acordo com Vieira et al. (2009). Para a testemunha foi utilizada água destilada. As cartelas foram colocadas sobre papel filtro para eliminação do excesso de umidade e secagem por uma hora. Posteriormente foram inseridas em tubos de vidro $(1,5 \times 6,0 \mathrm{~cm})$ com uma fêmea de $T$. pretiosum por 24 horas em câmara climatizada $\left(25 \pm 1^{\circ} \mathrm{C}, 70 \pm 10 \%\right.$ rh e14:10 L:D).

O parasitismo, avaliado por meio da contagem de ovos escuros do hospedeiro; a emergência, por meio da contagem dos ovos do hospedeiro que apresentavam orifício de saída dos adultos, vistos sob microscópio estereoscópio e, a razão sexual, calculada por $\mathrm{RS}=$ ( $\mathrm{n}^{\circ}$ de fêmeas $/ \mathrm{n}^{\circ}$ de adultos), sendo o sexo dos indivíduos determinado com base nas características morfológicas das antenas (Bowen \& Stern, 1966), foram os parâmetros avaliados. O parasitismo e a emergência foram corrigidos por Abbott (1925) $[(\mathrm{T}-\mathrm{t}) / \mathrm{T}]^{\star} 100$, onde: $\mathrm{T}=$ número de insetos vivos na parcela testemunha e $\mathrm{t}=$ número de insetos vivos na parcela tratada. Os produtos fitossanitários foram classificados com base na redução no parasitismo: classe 1- menor do que $30 \%$ de mortalidade, classe 2- entre 30 e $79 \%$, classe 3entre 80 e $99 \%$, classe $4-$ maior do que $99 \%$.

O delineamento experimental foi 0 inteiramente casualizado com 10 tratamentos e 25 repetições, sendo cada repetição representada por uma fêmea de $T$. pretiosum exposta à 40 ovos. As análises estatísticas foram feitas pelo programa Statgraphics Centurion XV version 15.1.02 (StatPoint $₫$ ). Os dados foram submetidos à análise de variância (ANOVA) e as médias comparadas pelo teste de Duncan $(p \leq 0,05)$. 


\section{RESULTADOS E DISCUSSÃO}

Parasitismo. Os produtos fitossanitários influenciaram o comportamento de parasitismo de adultos de $T$. pretiosum linhagem bonagota (Tabela 1). Tebufenozide e spirodiclofen causaram reduções inferiores a $20 \%$ no parasitismo (classe 1 ).

Reduções no parasitismo de $T$. pretiosum entre 63,8 e $79,7 \%$ foram observadas quando os ovos de $S$. cerealella foram tratados com abamectin, fenitrothion e methidathion (classe 2). Phosmet se enquadra como Classe 2, entretanto o impacto desse inseticida sobre o parasitismo foi pequeno em relação aos efeitos causados pelos três outros produtos fitossanitários dessa classe. Efeito negativo de phosmet foi $53,6 \%$ menor do que obtido por fenitrothion.

Carbaryl e malathion causaram o mesmo percentual de redução de parasitismo (98,1\%) (classe 3). Clorpyrifos foi o produto mais tóxico à $T$. pretiosum de acordo com a classificação adotada (classe 4), entretanto o efeito sobre o parasitismo não foi diferente do obtido por methidathion, carbaryl e malathion.

Emergência e razão sexual. A emergência de adultos de $T$. pretiosum linhagem bonagota foi afetada pelos produtos fitossanitários (Tabela 1). Dentre os inseticidas classificados na classe 3 e 4, carbaryl foi o único que reduziu significativamente a emergência em $50 \%$ de $T$. pretiosum. Clorpyrifós e malathion não tiveram efeito sobre a emergência, assim como tebufenozide, spirodiclofen phosmet e fenitrotion. Abamectin e methidathion apresentaram efeitos similares sobre a emergência, em média $20 \%$ de redução.

Não houve diferença entre os tratamentos para a razão sexual (Tabela 1), embora tenha sido observada um menor número de fêmeas no tratamento abamectin.

A realização de bioensaios visando determinar o impacto de produtos fitossanitários tem justificativa devido ao alto número de pulverizações em macieira no Brasil. Os produtos fitossanitários recomendados pela CTMIP (Protas \& Sanhueza 2004) foram selecionados através de referência científica por apresentarem menor impacto ambiental, entretanto poucos estudos sobre os efeitos destes produtos fitossanitários foram realizados para inimigos naturais coletados em macieira. De uma forma geral, há indícios que agrotóxicos prejudicam o parasitismo (Ballal et al. 2009), entretanto, parasitóides sofrem pressão de seleção.

Os estudos de seletividade com ovos colados em cartão e emergidos permitem que as fêmeas de Trichogramma spp. permaneçam em contato com os inseticidas como nas folhas de macieira. Além disso, os ovos também estão contaminados, o que faz com que a exposição seja maior e prolongada à medida que o parasitóide introduz o ovipositor no ovo. O efeito tóxico dos produtos fitos- sanitários aumenta devido ao maior contato do parasitóide com a superfície contaminada em relação as metodologias de pulverização sobre superfície inerte.

A baixa redução do parasitismo e emergência de $T$. pretiosum coletado em posturas $B$. salubricola causados por tebufenozide e spirodiclofen confirmaram estudos em fruteiras (Manzoni et al. 2006a, Giolo et al. 2007ac). Ambos produtos precisam ser ingeridos para atuarem no processo de ecdise e biosíntese de lipídeos, respectivamente, diferindo dos neurotóxicos que agem por contato. Phosmet mostrou-se seletivo aos adultos de T. pretiosum linhagem bonagota apesar de ser neurotóxico, normalmente de amplo espectro. Os três produtos não apresentaram nenhum efeito residual e não influenciaram a emergência.

Não se pode dizer o mesmo para abamectin, um recente acaricida do grupo químico lactones, derivado de um fungo do solo Streptomyces avermitilis e com ação no sistema nervoso. Além de reduzir o parasitismo, impediu $1 / 3$ das emergências devido a ação residual, situação similar ao ocorrido com methidation. Produtos fitossanitários com alto poder residual interferem na emergência quando Trichogramma spp. ingerem resíduos durante a abertura do orifício de emergência, como foi observado por Cônsoli et al. (2001). Os resultados de Manzoni et al. (2006a) mostraram efeitos mais drásticos de abamectin sobre o parasitismo de $T$. pretiosum coletado em pessegueiro. Apesar das diferenças metodológicas entre os estudos, os bioenssaios, com ovos imersos nos produtos fitossanitários, expõe os parasitoides ao contato direto com o residual dos ovos contaminados e aos vapores liberados pelos produtos fitossanitários (Silva \& Fay, 2004), ocorrendo uma maior taxa de intoxicação dos parasitóides (Rocha \& Carvalho 2004), o que não se observou.

Os efeitos de carbaryl, clorpyrifos e malathion diretamente sobre as fêmeas de $T$. pretiosum foram drásticos, pois não houve mais do que $1 \%$ de parasitismo. Carbaryl e malathion apresentaram efeito similar nos estudos de Giolo et al. (2005, 2007b) e Manzoni et al. (2006a, 2007). Em Fraiburgo, carbaryl é utilizado ao menos uma vez ao ano, pois é misturado ao ácido naftalenoacético (ANA) no raleio químico dos frutos (Camilo \& Paladini 2000) e malathion usado para o controle de moscadas-frutas, Anastrapha fraterculus (Diptera: Tephritidae). Clorpyrifos também foi considerado tóxico à outras linhagens de $T$. pretiosum (Cañete 2005, Hohmann, 1993, Delpuech \& Meyet 2003). É um dos inseticidas mais utilizados no controle de tortricídeos em macieiras em Fraiburgo, porém reduz a capacidade de parasitismo natural de $T$. pretiosum.

Abamectin, fenitrothion, methidathion e phosmet foram menos tóxicos a $T$. pretiosum linhagem bonagota coletada em macieira do que T. pretiosum coletado em pessegueiro (Manzoni et al. 
ARAÚJO, E.S. et al. Seletividade de inseticidas...

TABELA 1. Percentual de parasitismo (média \pm erro padrão), de emergência e razão sexual de Trichogramma pretiosum linhagem bonagota (Hymenoptera: trichogrammatidae) em ovos de Sitotroga cerealella (Lepidoptera: Gelechiidae) imersos em produtos fitossanitários recomendados pelo programa de Produção Integrada de Maçãs.

\begin{tabular}{|c|c|c|c|c|c|c|}
\hline Produto fitossanitário & $\begin{array}{l}\text { Parasitismo } \\
(\%)^{1}\end{array}$ & $\begin{array}{l}\text { Redução } \\
\text { do para- } \\
\text { sitismo } \\
(\%)\end{array}$ & $\begin{array}{l}\text { Emergência } \\
(\%)\end{array}$ & $\begin{array}{l}\text { Redução } \\
\text { da emer- } \\
\text { gência (\%) }\end{array}$ & Razão sexual & Classe $^{2}$ \\
\hline Tebufenozide & $45,2 \pm 4,73 a$ & 4,4 & $88,8 \pm 2,31 \mathrm{ab}$ & 0 & $0,5 \pm 0,07 a$ & 1 \\
\hline Spirodiclofen & $38,9 \pm 5,08 \mathrm{ab}$ & 17,8 & $88,0 \pm 2,60 a b$ & 0 & $0,5 \pm 0,07 a$ & 1 \\
\hline Phosmet & $31,1 \pm 5,12 b$ & 34,2 & $89,7 \pm 2,12 a b$ & 0 & $0,5 \pm 0,08 a$ & 2 \\
\hline Fenitrothion & $17,1 \pm 4,13 c$ & 63,8 & $96,7 \pm 1,31 \mathrm{a}$ & 0 & $0,7 \pm 0,04 a$ & 2 \\
\hline Abamectin & $15,0 \pm 2,36 c$ & 68,3 & $52,7 \pm 4,68 c$ & 27,3 & $0,4 \pm 0,08 a$ & 2 \\
\hline Methidathion & $9,6 \pm 2,29 \mathrm{~cd}$ & 79,7 & $63,2 \pm 7,23 \mathrm{~cd}$ & 12,8 & $0,8 \pm 0,06 a$ & 2 \\
\hline Carbaryl & $0,9 \pm 0,38 d$ & 98,1 & $50,0 \pm 1,00 \mathrm{e}$ & 31,1 & $0,7 \pm 0,07 a$ & 3 \\
\hline Malathion & $0,9 \pm 0,41 \mathrm{~d}$ & 98,1 & $100,0 \pm 0,00 a$ & 0 & $0,5 \pm 0,07 a$ & 3 \\
\hline Clorpyrifos & $0,3 \pm 0,22 \mathrm{~d}$ & 99,4 & $100,0 \pm 0,00 a$ & 0 & $0,8 \pm 0,07 a$ & 4 \\
\hline Testemunha & $47,3 \pm 4,43 a$ & - & $72,5 \pm 3,62 b c$ & - & $0,6 \pm 0,06 a$ & \\
\hline
\end{tabular}

${ }^{1}$ Médias seguidas pela mesma letra na coluna, não diferem estatisticamente entre si, pelo teste de Duncan ao nível de $5 \%$ de probabilidade.

${ }^{2}$ Classe 1 - menor do que $30 \%$ de mortalidade, classe 2- entre 30 e $79 \%$, classe 3 - entre 80 e $99 \%$, classe 4 - maior do que $99 \%$.

2006a, 2007; Giolo et al., 2007a). Resguardada as diferenças metodológicas entre os dois estudos, o fator associado as características genéticas entre as linhagens de parasitoide podem responder a essa diferença (Carvalho et al., 2002), em função da sua suscetibilidade interespecífica e intra-específica (Bull \& Coleman, 1985, Pratissoli et al., 2003). T. pretiosum linhagem bonagota foi coletado em um pomar comercial de macieira sob influencia dos produtos fitossanitários, enquanto que, T. pretiosum coletado em pessegueiro não foi exposto à methidathion e phosmet, pois estes não eram registrados em pessegueiro na época dos testes (Andrei, 2003). Além disso, a frequência de pulverização com organofosforados em macieira é maior do que em pessegueiro, resultando em seleção de populações dos parasitoides mais resistentes.

O parasitismo natural de $T$. pretiosum em ovos de $B$. salubricola linhagem bonagota ocorre durante todo o ano (Monteiro et al., 2004) e a eficiência do controle natural está sujeito à frequência de inseticidas para o controle de mariposa-oriental, lagarta-enroladeira-das-macieiras e moscas-dasfrutas. O uso repetitivo desses produtos fitossanitá- rios pode causar um efeito acumulativo na redução da população do parasitoide, em pomares de macieira, maior do que apresentado individualmente nesse estudo.

\section{CONCLUSÃO}

Os produtos fitossanitários tebufenozide e spirodiclofen são inócuos, enquanto que carbaryl, malathion e clorpyrifos são extremamente tóxicos à Trichogramma pretiosum linhagem bonagota.

A emergência de $T$. pretiosum linhagem bonagota é afetada quando em ovos de $S$. cerealella são tratados com carbaryl, methidation e abamectin.

A razão sexual de $T$. pretiosum linhagem bonagota não é afetada pelos produtos abamectin, carbaryl, clorpyrifos, fenitrothion, malathion, methidation, phosmet, tebufenozide e spirodiclofen.

\section{AGRADECIMENTOS}

À Associação dos Fruticultores de Fraiburgo pelo auxílio na criação de insetos. À UFPR e ao CNPq pela concessão de bolsas. 


\section{REFERÊNCIAS}

1.ABBOTT, W. S. A method of computing the effectiveness of an insecticide. Journal of Economic Entomology, Lanham, $v$ 18: 265-267, 1925.

2.ANDREI, E. Compêndio de defensivos agrícolas. $6^{\circ}$ edição, São Paulo, Andrei Editora, 2003,302 p.

3.BALLAL, C.R., SRINIVASAN R., JALALI S.K. Evaluation of an endosulfan tolerant strain of Trichogramma chilonis on cotton. BioControl, v. 54: 723-732, 2009.

4.BOWEN, W. R. \& STERN V.M. Effect of temperature on the production of males and sexual mosaics in a uniparental race of Trichogramma semifumatum (Hymenoptera: Trichogrammatidae). Annals Entomological Society American, Lexington, v. 59: 823-834, 1966.

5.BULL, D. L. \& COLEMAN R. J. Effects of pesticides on Trichogramma spp. Southwestern Entomologist, Dallas, v. 8: 156168, 1985.

6.CAMILO, A. P. \& PALLADINI L. A. Efeito de diferentes volumes de calda no raleio químico de frutos da macieira 'Gala' Pesquisa Agropecuária Brasileira, Brasília, v. 35: 2191-2195, 2000.

7.CAÑETE, C. L. Seletividade de inseticidas a espécies de Trichogramma (Hymenoptera: Trichogrammatidae). Tese de doutorado, Curitiba, UFPR, 106 p., 2005.

8.CARVALHO, G. A., REIS P. R., MORAES J. C., FUINI L. C., ROCHA L. C. D. \& GOUSSAIN M.M. Efeito de alguns inseticidas utilizados na cultura do tomateiro (Lycopersicon esculentum Mill.) a Trichogramma pretiosum Riley, 1879 (Hymenoptera: Trichogrammatidae). Ciência e Agrotecnologia, Lavras, v. 26: 1160-1166, 2002.

9.CÔNSOLI, F.L., BOTELHO P.S.M. \& PARRA J.R.P.. Selectivity of insecticides to the egg parasitoid Trichogramma galloi Zucchi, 1988, (Hym: Trichogrammatidae). Journal of Applied Entomology, v. 125: 37-43, 2001.

10.DELPUECH, J. M \& MEYRT J. Reduction in the sex ratio of the progeny of a parasitoid wasp (Trichogramma brassicae) surviving the insecticide clorpyrifos. Archives Environmental Contamination Toxicology, New York, v. 45: 203-208, 2003.

11.GIOLO, F. P.; GRUTZMACHER A. D.; MANZONI C. G.; LIMA C. A. B. \& NÖRNBERG S. D. Toxicidade de produtos fitossanitários utilizados na cultura do pessegueiro a adultos de Trichogramma pretiosum. Bragantia, Campinas, v. 66: 423431, 2007a.

12.GIOLO, F. P.; GRUTZMACHER A. D.; MANZONI C. G.; LIMA C. A. B.; HÄRTER W. R. \& MÜLLER C. Seletividade de produtos fitossanitários utilizados na cultura do pessegueiro a Trichogramma pretiosum Riley, 1879 (Hymenoptera: Trichogrammatidae). Ciência e Agrotecnologia, Lavras, v. 31: 1396-1401, 2007b.

13.GIOLO, F. P.; GRUTZMACHER A.D.; MANZONI S. D.; NÖRNBERG S. D. \& STEFANELLO G. J. Seletividade de agrotóxicos indicados na produção integrada de pêssego a T. pretiosum Riley, 1879 (Hymenoptera: Trichogrammatidae). Revista Brasileira de Fruticultura, Jaboticabal, v. 27: 222-225, 2005.

14.HASSAN, S. A. Seleção de espécies de Trichogramma para o uso em programas de controle biológico. p. 183-206. In: J.R.P. Parra \& R.A. Zucchi, Trichogramma e o controle biológico aplicado. Piracicaba, FEALQ, 1997,324 p.

15. HOHMANN, C. L. Efeito de alguns inseticidas sobre adultos de Trichogramma pretiosum Riley. Anais Sociedade Entomológica do Brasil, Piracicaba, v. 22: 563-568, 1993.

16.KOVALESKI, A. Pragas. In: Kovaleski. A. (ed.). Maçã: Fitossanidade. Brasília: Embrapa Informação Tecnológica, 85p. (Frutas do Brasil; 38), p. 10-33, 2004.

17.MANZONI, C. G.; GRUTZMACHER A. D.; GIOLO F. P.; LIMA C. A. B.; HARTER W. R. \& MULLER C. Seletividade de agrotóxicos usados na produção integrada de maçã para adultos de Trichogramma pretiosum. Pesquisa Agropecuária Brasileira, Brasília, v. 41: 1461-1467, 2006b.

18.MANZONI, C. G.; GRUTZMACHER A. D.; GIOLO F. P.; LIMA C. A. B.; HARTER W. R.; CASTILHOS R. V. \& PASCHOAL M. D. F. Seletividade de agroquímicos utilizados na Produção Integrada de Maçã aos parasitóides Trichogramma pretiosum Riley e Trichogramma atopovirilia Oatman \& Platner (Hymenoptera: Trichogrammatidae). BioAssay, Piracicaba, v. 2: 1-11, 2007.

19.MANZONI, C. G.; GRUTZMACHER A. D.; GIOLO F. P.; LIMA C. A. B.; NÖRNBERG S. D.; HARTER W. R. \& MULLER C. Seletividade de agrotóxicos recomendados na produção integrada da maçã a Trichogramma pretiosum Riley, 1879 (Hym.: Trichogrammatidae) em condições de laboratório. Revista Brasileira de Fruticultura, Jaboticabal, v. 28: 123-145, 2006a.

20.MONTEIRO, L. B. Inseticidas utilizados para o controle de moscas-das-frutas em pomares de macieira: ação sobre Neoseiulus californicus (Acari: Phytoseiidae). Scientia Agrária, Curitiba, v. 2: 81-85, 2001 a.

21.MONTEIRO, L. B. Seletividade de inseticidas a Neoseiulus californicus McGregor (Acari: Phytoseiidae) em macieira, no Rio Grande do Sul. Revista Brasileira de Fruticultura, Jaboticabal, v. 23: 589-592, 2001b.

22.MONTEIRO, L. B.; SOUZA A. \& BELLI E. L. Ocorrência de Trichogramma pretiosum Riley (Hymenoptera: Trichogrammatidae) em ovos de Bonagota cranaodes (Meyrick) (Lepidoptera: Tortricidae) em macieira. Revista Brasileira de Fruticultura, Jaboticabal, v. 26: 171-172, 2004.

23.NAVARRO, M. A. Trichogramma spp. Produción, uso y manejo en Colombia. Valle del Cauca, Guadalajara de Buga, 176 p., 1998

24.PASTORI, P. L.; ARIOLI C. J.; BOTTON M.; MONTEIRO L. B. \& MAFRA NETO A. Avaliação da técnica de disrupção sexual utilizando emissores SPLAT visando ao controle Bonagota salubricola (Meyrick) e Grapholita molesta (Busck) (Lepidoptera: Tortricidae) na pré-colheita de maçãs da cultivar Fuji. BioAssay, Piracicaba, v. 3: 1-8, 2008.

25.PASTORI, P. L.; MONTEIRO L. B.; BOTTON M. \& PRATISSOLI D. Capacidade de parasitismo de Trichogramma pretiosum Riley (Hymenoptera: Trichogrammatidae) em ovos de Bonagota salubricola (Meyrick) (Lepidoptera: Tortricidae) sob diferentes temperaturas. Neotropical Entomology, Piracicaba, v. 36: 926-937, 2007.

26.PRATISSOLI, D.; FORNAZIER M. J.; HOLTZ A. M; GONÇALVES J. R.; CHIORAMITAL A. B. \& ZAGO H. B.. Ocorrência de Trichogramma pretiosum em áreas comerciais de tomate, no Espírito Santo, em regiões de diferentes altitudes. Horticultura Brasileira, Campinas, v. 21: 73-76, 2003.

27.PROTAS, J. F. S. \& SANHUEZA R. M. V. Normas técnicas e documentos de acompanhamento da produção integrada de maçã - $2^{\circ}$ versão. Bento Gonçalves, RS, Embrapa Uva e Vinho, 80 p (Documentos, 33), 2004.

28.ROCHA, L. C. D. \& CARVALHO G. A. Adaptação da metodologia padrão IOBC para estudos de seletividade com Trichogramma pretiosum em condições de laboratório. Acta Scientiarum Agronomy, Maringá, v. 26: 315-320, 2004.

29.SILVA, C.M.S. \& FAY E.F. Agrotóxicos e ambiente. Brasília: Embrapa Informação Tecnológica, 2004. 400p.

30.VIANNA U. R., PRATISSOLI D., ZANUNCIO J.C., LIMA E.R., BRUNNER J., PEREIRA F.F., SERRÃO J.E. Insecticide toxicity to Trichogramma pretiosum (Hymenoptera: Trichogrammatidae) females and effect on descendant generation. Ecotoxicology, v. 18:180-186, 2009.

Scientia Agraria, Curitiba, v.14, n.1, p.35-39, Jan./Fev. 2013 